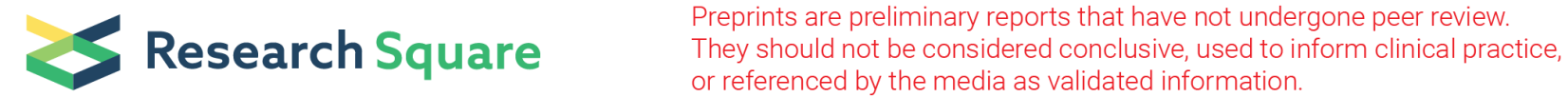

\section{Predictive value of monocyte to high-density lipoprotein ratio for the estimation of diabetes mellitus risk: Insights from a general population}

Hao Qian

China Medical University First Hospital https://orcid.org/0000-0002-1117-3498

Haoyu Wang

Chinese Academy of Medical Sciences \& Peking Union Medical College Fuwai Hospital

Zhi Du

China Medical University First Hospital

\section{Xiaofan Guo}

China Medical University First Hospital

Liqiang Zheng

China Medical University First Hospital

Zhao Li

China Medical University First Hospital

Hongmei Yang

China Medical University First Hospital

Shasha Yu

China Medical University First Hospital

Ying Zhou

China Medical University First Hospital

Yingxian Sun ( $\nabla$ yxsun@cmu.edu.cn )

\section{Research}

Keywords: Monocyte to high-density lipoprotein ratio, Diabetes, Epidemiology

Posted Date: April 23rd, 2020

DOI: https://doi.org/10.21203/rs.3.rs-21505/v1

License: (c) (i) This work is licensed under a Creative Commons Attribution 4.0 International License.

Read Full License 


\section{Abstract}

\section{Background}

Monocyte to high-density lipoprotein cholesterol ratio (MHR) is a novel inflammatory marker of inflammation and oxidative stress and has been used to predict multiple inflammation-related diseases, but data relative to diabetes mellitus are lacking. Hence, our study aimed to explore the impact of MHR on diabetes and its value to improve the risk stratification and prevention of diabetes in a large cohort of general Chinese population.

\section{Method}

This study selected 8142 (males: $45.76 \%$ ) participants who participated in the rural cardiovascular health research in Northeast China between 2012 and 2013. MHR was defined as blood monocyte count ratio to high-density lipoprotein cholesterol concentration

\section{Results}

The prevalence of diabetes is $10.7 \%$. By correcting for potential confounding factors, per SD increase of MHR caused a $11.2 \%$ additional risk for diabetes, in addition, the top quartile of MHR increased the risk of diabetes by $33.1 \%$ over the bottom quartile.

Furthermore, smooth curve fitting showed a linear and strong correlation between MHR and diabetes. Finally, Category-free net reclassification improvement $(0.085,95 \% \mathrm{Cl}: 80.016-0.155, \mathrm{P}=0.016)$ and integrated discrimination improvement $(0.001,95 \% \mathrm{Cl}: 0.000-0.0024, \mathrm{P}=0.018)$ also demonstrated significant improvements

\section{Conclusions}

Our study revealed an independent and robust correlation between MHR and prevalent diabetes mellitus in general population. Furthermore, our results suggest the importance of simultaneous inflammation and lipids control in the prevention of diabetes mellitus, highlighting the value of MHR to optimize the risk stratification and prevention of diabetes mellitus. These findings have implications for the prevention of diabetes and its adverse outcomes.

\section{Introduction}

Diabetes is a group of metabolic diseases, which are caused by long-term hyperglycemia due to impaired insulin secretion or its biological effects, and the coexistence of both. Diabetes can affect multiple tissues and organs, especially chronic damage to the eyes, kidneys, heart, blood vessels, and nerves, and dysfunction[1, 2]. China has one of the fastest rising rates of diabetes. Latest epidemiological studies show that about $11 \%$ of the population has diabetes[3], and only about $30 \%$ of them were treated[4]. However, in remote areas of China, the incidence of many diabetes is often neglected, leading to a large 
proportion of complications caused by diabetes and deaths due to diabetes[5]. To begin to determine the early diagnosis of diabetes in remote areas of China, early treatment is particularly important. According to the existing literature, the risk factors of diabetes mainly include: sex, age, family history of diabetes, less than college education, overweight, obesity, central obesity, raised SBP [6-8].

High-density lipoprotein (HDL) is mainly secreted by the liver and small intestine and plays an important role in the reverse transport of cholesterol (RCT)[9]. HDL has anti-inflammatory and anti-atherosclerotic effects[10, 11], It is a "lipid scavenger" in blood vessels. In the cardiovascular system, low-level highdensity lipoprotein, cholesterol (HDL-C) dyslipidemia is strongly negatively correlated with the risk of cardiovascular disease[12], it is one of the important indicators to predict the incidence of cardiovascular disease. It has also been reported that high-density lipoprotein can promote glucose-stimulated insulin secretion and exert an anti-diabetic effect. At the same time, HDL have also been found to stimulate insulin-dependent and insulin-independent glucose uptake into skeletal muscle, adipose tissue, and liver[13]. Increasing incidence of microvascular and macrovascular complications in diabetic patients, related to dyslipidemia, endothelium-dependent vasodilatation, atherosclerosis, and inflammation[1416]. In recent years, many HDL analogues have been used in the treatment of diabete[17].

Monocytes are mononuclear myeloid cells that develop in the bone marrow and circulate within the bloodstream[18]. It is currently considered to be a precursor to macrophages and dendritic cells[19, 20]. The main function of monocytes is to provide innate immune defense and prevent infection and damage. They are an important part of the body's defense system, but they are also involved in many central nervous system (CNS) diseases[21]. When inflammation occurs, a large number of monocytes are quickly mobilized to the site of inflammation throughout the body, where they act as a special "emergency team" [22]. It has been reported that monocytes are related to the angiogenesis of atherosclerosis[23]. In type 2 diabetes, the number of monocytes is up-regulated, and the imbalance of proinflammatory cytokines and anti-inflammatory cytokines affects the function of the immune system, which may be the cause and result of type 2 diabetes[24]. Long-term functional reprogramming of monocytes and macrophages induced by hyperglycemia plays an important role in the occurrence of cardiovascular complications in patients with diabetes[25].

From these observations, a model constructed using these two indices, that is, the emerging monocyte-toHDL-C ratio (MHR), has been put forward as a simple surrogate of IR inflammation. Additionally, previous studies have found that MHR has the capacity to predict a variety of inflammation-related diseases. The association between MHR and diabetes remains unknown. Hence, our present study aimed to investigate the effect of MHR on the prevalent diabetes and assess the value of MHR to optimize the risk stratification and prevention of diabetes based on a general population.

\section{Materials And Methods \\ Study population}


This study is based on a large population-based epidemiological cross-sectional study named Northeast China Rural Cardiovascular Health Study (NCRCHS), which was performed between January and August 2013 , data of 11,956 permanent residents (age $\geq 35$ years) in rural areas of the northeastern China were collected. Subjects with pregnancy, malignancy or mental disorders were excluded from the study. The full details of the research design and theoretical basis of this part are extensively described elsewhere[26-28]. In the present study, 3814 participants were excluded for missing biochemical and clinical data. Finally, the study cohort was composed of 8142 subjects (Fig. 1). Our research plan approved by The Ethics Committee of China and China Medical University. Each participant has completed relevant informed consent.

\section{Data Collection \& Measurements}

Our previous research has reported on the process of data collection and measurement as they participated in the same surveys as this study[26-28]. We conducted the necessary professional training for cardiologists and nurses before the study began and passed the relevant tests.

Information about demographic data, health-related behaviors, anthropometric parameters, family annual income level, education level, current smoking, current drinking, and family history of diabetes was collected through a questionnaire survey by trained medical staff. Quality control was conducted by a central steering committee with a subcommittee. Race was divided into 2 groups: Han and others. Family history of DM was defined as anyone of the participant's family members (including father, mother, siblings and children) had diabetes. Education level was regarded as three ordinal groups: primary school or below, middle school, high school or above. Three ordinal groups ( $\leq 5000 \mathrm{CNY}, 5000-20,000 \mathrm{CNY}$,> $20,000 \mathrm{CNY}$ ) were utilized to represent the family annual income level. Collect information on current smoking and drinking conditions through a series of questions about participants' smoking and drinking history.

After the subjects had sat and relaxed for at least 5 minutes, blood pressure was measured by 2 randomly selected trained medical staff, recording 3 consecutive readings of each subject and calculating the average value for statistical analysis.

As for the measurement of anthropometric indicators, participants were asked to wear light clothes without shoes. The calibrated digital scale is used to quantify the standard weight to the nearest $0.1 \mathrm{~kg}$. The calibrated tachymeter was used to quantify the standard height to the nearest $0.1 \mathrm{~cm}$, and the subjects remained standing.

Our previous research report described the storage process and laboratory measurement methods[2628]. In general, We collected blood samples by fasting ( 12 hours) through venipuncture using an EDTA tubes. Plasma is separated and stored at $-20^{\circ} \mathrm{C}$. The whole process is completed in 1 hour. The samples were analyzed biochemically using an Olympus AU640 automatic analyzer (Olympus, Kobe, Japan). The 
samples were analyzed biochemically using an Olympus AU640 automatic analyzer (Olympus, Kobe, Japan).

\section{Definitions}

Body mass index (BMI) was calculated as mean weight divided by mean height squared $(\mathrm{kg} / \mathrm{m} 2)$ [29]. MHR was defined as blood monocyte count ratio to high-density lipoprotein cholesterol concentration[30].

Hypertension is defined as systolic blood pressure (SBP) $\geq 140 \mathrm{mmHg}$ and /or diastolic blood pressure (DBP) $\geq 90 \mathrm{mmHg}$ [31]. At the same time if the subject self-reports that the subject's previous medical history was diagnosed as high blood pressure, or is currently taking antihypertensive drugs were also considered to be hypertensive.

The determination of diabetes wsa based on fasting plasma glucose (FPG) $\geq 7.0 \mathrm{mmol} / \mathrm{L}$ and/or subjects had been previously diagnosed with diabetes in subject self-report[32]. In addition, subjects who received hypoglycemic treatment at baseline were also considered to be diabetic.

\section{Statistical Analyses}

Each gender was analyzed separately. If the continuous variable has a normal distribution, it is expressed as the mean \pm standard deviation (SD), and if it does not have a normal distribution, it is expressed as the median (interquartile range). Categorical variables are expressed by frequency (percentage). Prior to inference analysis, MHR values were log. The Student's t test (normal distribution) or Mann-Whitney test (non-normal distribution) were used to compare the diabetic group with the non-diabetic group to show the difference in continuous variables. Chi-square test was employed to compare categorical variables between groups. Besides, in order to compare ordinal categorical variables (education level, annual household income, and physical activity) between groups, a rank sum test was presented to use ordinal information. A multivariate logistic regression model was performed to correct potential confounding covariates to determine the adjusted odds ratio (ORS) and 95\% confidence interval (CIS) of the independent association between MHR and diabetes. The confounding variables variables for adjustment include age, sex, education level, family annual income level, physical activity, current smoking, current drinking, family history of diabetes, BMI and SBP. The analysis was performed with MHR as a continuous variable (increase per SD), and then classified into quartiles. The results are shown as odds ratios (ORs) and $95 \%$ confidence intervals $(95 \% \mathrm{Cl})$. A generalized additive model function with spline smoothing is used to examine MHR and the risk of diabetes. We also utilized receiver operating characteristic (ROC) curve, integrated discrimination improvement (IDI) and category-free net reclas sification improvement (NRI) to estimate the potential of MHR to enhance the risk of diabetes. All data analyzed by SPSS 25.0 software (IBP corp), Empower Stats (http://www.empowerstats.com, X\&Y Solutions, Inc., Boston, MA) and statistical software packages R (http://www.R-project.org, The R Foundation). A two-tailed $P$ value of less than 0.05 was considered significant. 


\section{Results}

After excluding subjects with missing data, a total of 8142 participants participated in this analysis. Table 1 summarizes the data of 8142 eligible subjects (males: $45.76 \%$ ). In this study population, the prevalence of diabetes was $10.7 \%$. Based on demographic data, Subjects with diabetes had lower education levels than normal subjects. Unexpectedly, middle-income population has more subjects with diabetes than low- and high-income populations and moderately physical active subjects had a higher rate of diabetes. In addition, diabetes subjects are not significantly related to current smoking and current drinking. The anthropometric characteristics indicate greater BMI in the diabetic group. Concordantly, Many subjects had diabetes and hypertension at the same time, their SBP and DBP increased significantly. As expected, subjects with diabetes have a family history of diabetes. Through blood biochemical examination we found that the levels of TC, TG, and LDL-C in patients with diabetes were significantly higher than those in the control group, while the HDL-C level was greatly reduced in diabetes. Correspondingly, the white blood cell count, neutrophil and monocyte counts increased in diabetic patients. Finally, the MHR level of diabetic patients was higher than that of the control group. 
Table 1

Characteristics of subjects stratified by diabetes

\begin{tabular}{|c|c|c|c|}
\hline Variables & Diabetes(N = 872) & Non-diabetes $(\mathrm{N}=7270)$ & $P$ value \\
\hline Age (years) & $57.99 \pm 9.67$ & $53.66 \pm 10.48$ & $<0.001$ \\
\hline Male (\%) & $382(43.8 \%)$ & $3344(46 \%)$ & 0.22 \\
\hline Education level (\%) & & & $<0.001$ \\
\hline Primary school or below & $544(62.4 \%)$ & $3765(51.8 \%)$ & \\
\hline Middle school & $256(29.4 \%)$ & $2875((39.5 \%)$ & \\
\hline High school or above & $72((8.3 \%)$ & $702(8.7 \%)$ & \\
\hline Income (CNY) (\%) & & & 0.238 \\
\hline$\leq 5000$ & $102(11.7 \%)$ & $752(10.3 \%)$ & \\
\hline $5000-20,000$ & $467(53.6 \%)$ & $3810(52.4 \%)$ & \\
\hline$>20,000$ & $303(34.7 \%)$ & $2708(37.2)$ & \\
\hline Physical activity (\%) & & & $<0.001$ \\
\hline Low & $350(40.1 \%)$ & $2184(30.0 \%)$ & \\
\hline Middle & $459(52.6 \%)$ & $4631(63.7 \%)$ & \\
\hline High & $63(7.2 \%)$ & $455(6.3 \%)$ & \\
\hline Family history of diabetes & $190(21.8 \%)$ & $557(7.7 \%)$ & $<0.001$ \\
\hline Hypertension (\%) & $632(72.5 \%)$ & $3176(43.7 \%)$ & $<0.001$ \\
\hline Current smoking (\%) & $263(30.2 \%)$ & $2773(34.5 \%)$ & 0.1 \\
\hline Current drinking (\%) & $182(20.9 \%)$ & $1562(21.5 \%)$ & 0.676 \\
\hline $\mathrm{BMI}\left(\mathrm{kg} / \mathrm{m}^{2}\right)$ & $25.94 \pm 3.62$ & $24.37 \pm 3.57$ & $<0.001$ \\
\hline
\end{tabular}

Data are expressed as mean \pm standard deviation (SD) or median (interquartile range) and numbers (percentage) as appropriate

Abbreviations: CNY Chinese currency ( $1 \mathrm{CNY}=0.15$ USD), BMI body mass index, SBP systolic blood pressure, DBP diastolic blood

pressure, TC total cholesterol, TG triglyceride, HDL-C high-density lipoprotein cholesterol, LDL-C lowdensity lipoprotein cholesterol,

WBC white Blood. MHR monocyte count to high-density lipoprotein ratio.

Comparisons of category variables between groups were tested by chi-square test or rank-sum test (ordinal category variables) were tested by Student's t or Mann-Whitney test. 


\begin{tabular}{|c|c|c|c|}
\hline Variables & Diabetes(N = 872) & Non-diabetes $(\mathrm{N}=7270)$ & $P$ value \\
\hline Mean SBP (mmHg) & $150.69 \pm 23.24$ & $137.23 \pm 21.34$ & $<0.001$ \\
\hline Mean DBP $(\mathrm{mmHg})$ & $85.52 \pm 12.56$ & $81.43 \pm 11.44$ & $<0.001$ \\
\hline $\mathrm{TC}(\mathrm{mmol} / \mathrm{L})$ & $5.74 \pm 1.28$ & $5.27 \pm 1.07$ & $<0.001$ \\
\hline TG (mmol/L) & $2.52 \pm 2.43$ & $1.58 \pm 1.28$ & $<0.001$ \\
\hline $\mathrm{HDL}-\mathrm{C}(\mathrm{mmol} / \mathrm{L})$ & $1.28 \pm 0.31$ & $1.35 \pm 0.32$ & $<0.001$ \\
\hline LDL-C (mmol/L) & $3.16 \pm 0,92$ & $2.86 \pm 0.78$ & $<0.001$ \\
\hline Neutrophil count $\left(10^{9} / \mathrm{L}\right)$ & $4.28 \pm 4.28$ & $3.69 \pm 2.56$ & $<0.001$ \\
\hline Lymphocyte count $\left(10^{9} / \mathrm{L}\right)$ & $2.56 \pm 3.85$ & $2.18 \pm 2.53$ & $<0.001$ \\
\hline Monocyte count $\left(10^{9} / \mathrm{L}\right)$ & $0.52 \pm 0.32$ & $0.48 \pm 0.28$ & $<0.001$ \\
\hline MHR & $0.43 \pm 0.28$ & $0.38 \pm 0.24$ & $<0.001$ \\
\hline \multicolumn{4}{|c|}{$\begin{array}{l}\text { Data are expressed as mean } \pm \text { standard deviation (SD) or median (interquartile range) and numbers } \\
\text { (percentage) as appropriate }\end{array}$} \\
\hline \multicolumn{4}{|c|}{$\begin{array}{l}\text { Abbreviations: CNY Chinese currency }(1 \mathrm{CNY}=0.15 \text { USD), BMI body mass index, SBP systolic blood } \\
\text { pressure, DBP diastolic blood }\end{array}$} \\
\hline \multicolumn{4}{|c|}{$\begin{array}{l}\text { pressure, TC total cholesterol, TG triglyceride, HDL-C high-density lipoprotein cholesterol, LDL-C low- } \\
\text { density lipoprotein cholesterol, }\end{array}$} \\
\hline \multicolumn{4}{|c|}{ WBC white Blood. MHR monocyte count to high-density lipoprotein ratio. } \\
\hline $\begin{array}{l}\text { Comparisons of category va } \\
\text { (ordinal category variables) }\end{array}$ & $\begin{array}{l}\text { between groups } \mathrm{n} \\
\text { sted by Student's } \mathrm{t}\end{array}$ & $\begin{array}{l}\text { ed by chi-square test or } \\
\text { In-Whitney test. }\end{array}$ & m test \\
\hline
\end{tabular}

Multivariate logistic regression model demonstrated the association between MHR and diabetes (Table 2). We first adjusted age and gender, each SD increment of MHR could cast additional $17.5 \%$ risk of diabetes on subjects. Then, We had made corresponding adjustments to education level, family annual income level, physical activity, current smoking, current drinking, family history of diabetes, BMI and SBP, each SD increment of MHR could cast additional $11.2 \%$ risk of subjects with diabetes. When we divide MHR into quartiles, we observed a $33.1 \%$ increase of the risk for diabetes when comparing top quartile with bottom category in the adjusted model. Furthermore, the risk of diabetes displayed an increasing trend across the quartiles ( $P$ for trend $\triangle 0.001$ ). To further explore the dose-response relationship between MHR and the risk of diabetes, We applied smooth curve fitting with full adjustment of all covariates (Fig. 2). The curve demonstrated a linear correlation between normalized MHR and the risk of diabetes. This result was consistent with the quartile analysis of logistic regression. 
Table 2

Evaluation of the impact of MHR on diabetes by multivariate logistic regression models

$\begin{array}{ll}\text { Variables } & \text { Odds Ratio } \\ (95 \% \mathrm{Cl})\end{array}$

\begin{tabular}{|c|c|c|c|c|c|c|}
\hline & Crude & $\begin{array}{l}P \\
\text { value }\end{array}$ & Model 1 & $\begin{array}{l}\mathrm{P} \\
\text { value }\end{array}$ & Model 2 & $\begin{array}{l}P \\
\text { value }\end{array}$ \\
\hline $\begin{array}{l}\text { MHR (Per } 1 \text { SD } \\
\text { increase) }\end{array}$ & $\begin{array}{l}\text { 1.178(1.108, } \\
1.253)\end{array}$ & $\varangle 0.001$ & $\begin{array}{l}1.175(1.104 \\
1.251)\end{array}$ & $<.001$ & $\begin{array}{l}1.112(1.042, \\
1.187)\end{array}$ & 0.001 \\
\hline
\end{tabular}

Quartiles of MHR

$\begin{array}{ll}\text { Quartile } 1 & \begin{array}{l}1.000 \\ \text { (reference) }\end{array}\end{array}$

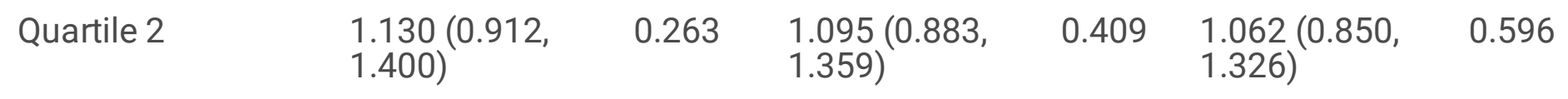

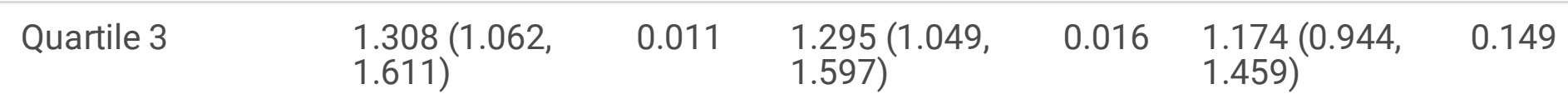

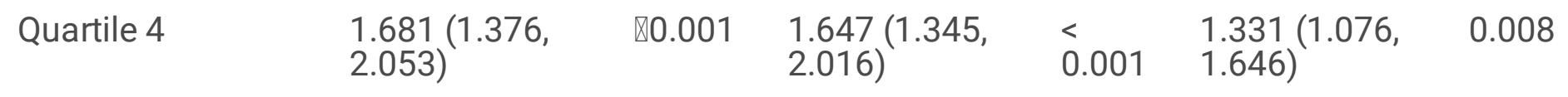

P for trend $\llbracket 0.001$

$<$ 0.001 $\llbracket 0.001$

Abbreviations: MHR monocyte count to high-density lipoprotein cholesterol ratio, OR odds ratio; $95 \%$ Cl: 95\% confidence interval, SD standard deviation. Other abbreviations as in Table 1

Crude: no adjustment;

Model 1: adjusted for age, sex;

Model 2: adjusted for age, sex, education level, family annual income level, physical activity, current smoking, current drinking, family history of diabetes, body mass index and systolic blood pressure.

Quartile 1: MHR < 0.229; Quartile 2: $0.229 \leq$ MHR < 0.341; Quartile 3: $0.341 \leq$ MHR < 0.485; Quartile 4:

$\mathrm{MHR} \geq 0.485$

Table 3

Comparison of the risk stratifying ability of MHR in addition to clinical risk factors

\begin{tabular}{|lllll|}
\hline Model & NRI (category free) & P value & IDI & P value \\
\hline Clinical risk factors ${ }^{\mathrm{a}}$ & Reference & Reference & Reference & Reference \\
\hline Clinical risk factors + MHR & $0.085(0.016,0.155)$ & 0.016 & $0.001(0.000,0.0024)$ & 0.018 \\
\hline $\begin{array}{l}\text { C-Statistic, net reclassification improvement (NRI) and integrated discrimination improvement (IDI) } \\
\text { were compared between models. The reference model included risk factors only, including age, sex, } \\
\text { physical activity, current smoking, current drinking, body mass index and systolic blood pressure. }\end{array}$ \\
\hline
\end{tabular}


Finally, we performed ROC analysis and reclassification analysis to explore the value of MHR to improve the risk stratification of diabetes. When adding MHR into established clinical risk factors (including age, sex, education level, family annual income level, physical activity, current smoking, current drinking, family history of diabetes, BMI and SBP), we failed to find a significant improvement in the AUC. However, both category free NRI $(0.085,95 \% \mathrm{Cl}: 0.016-0.155, \mathrm{P}=0.016)$ and IDI $(0.001,95 \% \mathrm{Cl}: 0.000-0.024, \mathrm{P}=$ 0.018 ) showed a significant advancement to stratify the risk of diabetes when adding MHR into clinical risk factors.

\section{Discussion}

Based on the population in Northeast China's rural areas, we for the first time discovered the significant association between MHR and the risk of diabetes in a general population. Furthermore, our work demonstrated the association was linear in the whole range of MHR, suggesting the linearity of MHR to indicate the presence of diabetes mellitus. Additionally, our results implicate the potential usefulness of MHR to refine the risk stratification of diabetes when introducing MHR into several established clinical risk factors. Our work also provides clinical evidence for the value of simultaneous inflammation and lipid control to prevent diabetes. More importantly, these findings implicate the value of MHR to optimize the risk stratification and prevention of diabetes mellitus.

MHR as a new circulating biomarker, is used to predict indicators of inflammation and dyslipidemia. At the same time, MHR has also been reported as a new prognostic indicator of cardiovascular disease. Epidemiological evidence suggests that high concentrations of plasma HDL have a protective effect on the cardiovascular system. HDL can prevent and reverse the activation of monocytes, which is of great significance for the treatment of many inflammatory diseases [33]. HDL can also protect endothelial cells from the adverse effects of LDL and prevent LDL oxidation [34,35]. Existing evidence suggests that $\mathrm{HDL}$ conversion appears to be accelerated in patients with diabetes, and low concentrations of HDL cholesterol in plasma are the result of increased conversion rates. The higher the plasma insulin level in these patients, the lower the plasma HDL cholesterol concentration [36].

The immune response is a complex system composed of cells and humoral components. They have the ability to detect non-self structures and resist invasion of pathogens [37]. Dendritic cells initiate and regulate a highly pathogen-specific adaptive immune response and are essential for the development of immune memory and tolerance [38]. Dendritic cells recognize pathogens and deliver antigens to initiate antigen-specific adaptive immune responses, thereby connecting the innate and adaptive immune systems [39]. Monocytes and macrophages are phagocytic cells, they belong to myeloid cell lineage and play a key role in the innate immune response to pathogens [40]. Under various inflammatory conditions, monocytes circulate through the blood, recruit and extravasate into inflamed tissues. involving rolling, adhesion, and transmigration. Whether it is infection or aseptic injury, monocyte recruitment is a key feature [41]. 
Obesity is considered a low-level systemic inflammatory state, in adipose tissue, monocytes migrate to adipose tissue and become macrophages, and macrophage aggregation is an obvious feature of obesity $[42,43]$. In obesity, adipocytes release pro-inflammatory cytokines and fatty acids to induce typical activation of macrophages [44]. In addition to proinflammatory cytokines, which promote adipoinflammation and lead to insulin resistance, macrophages can also release an adipokine that causes insulin resistance [45]. The increase of HDL level can prevent the formation of bone marrow, monocyte and neutrophil induced by diabetes, and reduce the cardiovascular risk of diabetic patients [46]. More and more evidences show that the increase of monocytes caused by diabetes aggravates the process of cardiovascular disease, especially atherosclerosis [47-49]. Whether monocyte and macrophage changes are caused by altered glucose levels, insulin deficiency or insulin resistance, lipid abnormalities, or a combination of these factors is still debated.

It is generally known that atherosclerosis is a chronic, non-resolving, low-grade sterile inflammation of the arterial wall, macrophages have been considered a major factor in atherosclerosis and its thrombotic complications for the past two decades [50]. The first step in the formation of atherosclerosis is the exposure of vascular cells to excess lipid (LDL), accompanied by activation / dysfunction of endothelial cells and internalization and deposition of intimal lipids. LDL enhances a series of pro-inflammatory responses that enable the activation, recruitment, and migration of different immune cells (monocytes, neutrophils, natural killer cells, and dendritic cells), most important is the contribution of circulating monocytes [51, 52]. Macrophage inflammation leads to increased oxidative stress and increased secretion of cytokines / chemokines, more LDL oxidation, endothelial cell activation and monocyte recruitment, which further engulf oxidized LDL-C to form foam cells [53]. In the late stages, matrixdegraded proteases and cytokines secreted by macrophages cause thinning of the fibrous caps that prevent blood from contacting the prethrombotic material in the plaque. Eventually, the cap will disintegrate, and subsequent plaque fragments will release tissue factors into the blood. Thrombosis can cause acute arterial stenosis, such as coronary syndrome, myocardial infarction, and stroke [54, 55].

We observe that whether MHR is a continuous or categorical variable, there is a positive correlation between MHR and the incidence of diabetes, which is consistent with our hypothesis. In the multivariate adjusted model, higher MHR levels were significantly associated with the incidence of diabetes. We further demonstrated that the linear association of MHR with diabetes through smooth curve fitting analysis. When using MHR as an indicator of the risk of diabetes, a higher MHR value means a higher risk of diabetes, and there is no threshold or saturation effect for this association. A recent study shows that MHR levels are linked to smoking [56], There are also reports in the literature that smoking is linked to diabetes [57]. However, in our data, the strong correlation between elevated MHR and diabetes was independent current smoking status. This finding confirms the stability of MHR on stratification of diabetes risk. In addition, this association persisted in the stratified analysis of gender, age, SBP, and BMI, suggesting that the risk stratification ability of MHR is applicable to a wide range of subjects.

We applied ROC and reclassification analysis to evaluate the risk stratification ability of MHR for diabetes. We added clinical risk factors (including gender, age, current smoking and drinking status, SBP, 
and $\mathrm{BMI}$ ) to the model and significantly improved stroke recognition. In order to more accurately confirm the ability of MHR to optimize the stratification of diabetes risk, we conducted a reclassification analysis including IDI and NRI [58]. When MHR was introduced into the established risk factor model, the results of category-free NRI and IDI both significantly improved the stratification of diabetes risk. In summary, our results suggest that MHR has the ability to optimize the risk stratification of diabetes (Table 2).

We acknowledge that our research has certain limitations, first, due to the cross-sectional design of our study, we can only suggest a correlation between MHR and diabetes, and we cannot draw any causal conclusions about the temporal relationship of MHR with diabetes. Secondly, This study represents the general population in rural areas of Northeast China, but its applicability to the general population in China is still worth more research to assess. Third, We adjusted as much confounding variables as possible, but the presence of unmeasured confounding variables may affect the results. Finally, residual confounding caused by uncollected or unknown risk factors can introduce bias into our results.

\section{Conclusions}

In summary, MHR is independently associated with diabetes in rual Chinese population, and shows incremental predictive value relative to traditional clinical risk factors. In addition, our results suggest the necessity of simultaneous inflammation and lipids control in the prevention of diabetes. Most importantly, our results also suggest that MHR is of great value in optimizing the risk stratification of diabetes. pointing out a simple and cost-effective solution to alleviate the burden of diabetes.

\section{Abbreviations}

BMI: Body mass index; Cl: Confidence interval; CNY: Chinese yuan, 1CNY = 0.15USD; DBP: Diastolic blood pressure; FPG: Fasting plasma glucose; HDL-C: High-density lipoprotein cholesterol; IDI: Integrated discrimination improvement; LDL-C: Low-density lipoprotein cholesterol; MHR: Monocyte count to highdensity lipoprotein cholesterol ratio; NCRCHS: Northeast China Rural Cardiovascular Health Study; NRI: Net reclassification improvement; OR: Odds ratio; ROC: Receiver operating characteristic curve; SBP: Systolic blood.

\section{Declarations}

\section{Acknowledgements}

We would like to express our gratitude to all those who exert their effects in achieving this study.

\section{Authors' contributions}

In this study, HYW, HQ, and ZD did the study design, statistical analyses and results interpretation. ZD participated as analyzing and resolving difficulties of analytic strategies and results discussion. Finally, 
HYW, HQ functioned as final reviewer and corresponding author. All authors read and approved the final manuscript.

\section{Funding}

This research was supported by funds from the National Key Research and Development Program from the Ministry of Science and Technology of China (Project Grant \# 2018YFC1312400, Sub-project Grant \# 2018YFC1312403) and National Natural Science Foundation of China (Grant \# 81800361), and also supported by grants from "Thirteenth Five-Year" program funds (The National Key Research and Development Program of China, Grant \#2017YFC1307600).

\section{Availability of data and materials}

The datasets used and/or analyzed during the current study are available from the corresponding author on reasonable request.

\section{Ethics approval and consent to participate}

This study was conducted in compliance with the ethical principle of the Declaration of Helsinki. All participants provided written informed consent and all procedures were performed in accordance with the ethical standards. The study protocol was approved by the Ethics Committee of China Medical University (Shenyang, China).

\section{Consent for publication}

All co-authors and participants have given their consent for publication of this article in Lipids in Health and Disease.

\section{Competing interests}

The authors declare that they have no competing interests.

\section{Footnotes}

Publisher's Note

Springer Nature remains neutral with regard to jurisdictional claims in published maps and institutional affiliations.Hao Qian,Hao-Yu Wang contributed equally to this work. Zhi Du , Xiao-Fan Guo , Li-Qiang Zheng , Zhao Li , Hong-Mei Yang , Sha-Sha Yu ,Ying Li, they guided the study and made valuable suggestions

\section{Contributor Information}

Hao Qian, Email: hqian@cmu.edu.cn 
Hao-Yu Wang, Email: dallashaoyu@163.com

Zhi Du, Email: zdu@cmu.edu.cn

Xiao-Fan Guo, Email: guoxiaofan1986@hotmail.com

Li-Qiang Zheng, Email: liqiangzheng@126.com

Zhao Li, Email: 2680481161@qq.com

Hong-Mei Yang, Email: eileen8222@163.com

Sha-Sha Yu, Email: cmu1h@outlook.com

Ying Zhou, Email: zhouying8111003@126.com

Ying-Xian Sun, Phone: +86 13804068899, Email: yxsun@cmu.edu.cn

\section{References}

1. American Diabetes A. Diagnosis and classification of diabetes mellitus. Diabetes Care. 2013;36(Suppl 1):67-74.

2. Skyler JS, Cahill GF Jr. Diabetes mellitus: progress and directions. Am J Med. 1981;70(1):101-4.

3. Ma RCW. Epidemiology of diabetes and diabetic complications in China. Diabetologia 2018, 61(6):1249-1260.

4. Wang L, Gao P, Zhang M, Huang Z, Zhang D, Deng Q, et al. Prevalence and Ethnic Pattern of Diabetes and Prediabetes in China in 2013. JAMA 2017, 317(24):2515-2523.

5. Bragg F, Holmes MV, Iona A, Guo Y, Du H, Chen Y, et al. Association Between Diabetes and CauseSpecific Mortality in Rural and Urban Areas of China. JAMA 2017, 317(3):280-289.

6. Yang W, Lu J, Weng J, Jia W, Ji L, Xiao J, et al. Prevalence of diabetes among men and women in China. N Engl J Med. 2010;362(12):1090-101.

7. Zuo H, Shi Z, Hussain A. Prevalence, trends and risk factors for the diabetes epidemic in China: a systematic review and meta-analysis. Diabetes Res Clin Pract. 2014;104(1):63-72.

8. Xu Y, Wang L, He J, Bi Y, Li M, Wang T, et al. Prevalence and control of diabetes in Chinese adults. JAMA. 2013;310(9):948-59.

9. Zhou L, Li C, Gao L, Wang A. High-density lipoprotein synthesis and metabolism (Review). Mol Med Rep. 2015;12(3):4015-21.

10. Rosenson RS, Brewer HB Jr, Chapman MJ, Fazio S, Hussain MM, Kontush A, et al. HDL measures, particle heterogeneity, proposed nomenclature, and relation to atherosclerotic cardiovascular events. Clin Chem. 2011;57(3):392-410. 
11. Hu J, Xi D, Zhao J, Luo T, Liu J, Lu H, et al. High-density Lipoprotein and Inflammation and Its Significance to Atherosclerosis. Am J Med Sci. 2016;352(4):408-15.

12. He Y, Kothari V, Bornfeldt KE. High-Density Lipoprotein Function in Cardiovascular Disease and Diabetes Mellitus. Arterioscler Thromb Vasc Biol. 2018;38(2):e10-6.

13. von Eckardstein A, Widmann C. High-density lipoprotein, beta cells, and diabetes. Cardiovasc Res. 2014;103(3):384-94.

14. Kibel A, Selthofer-Relatic K, Drenjancevic I, Bacun T, Bosnjak I, Kibel D, et al. Coronary microvascular dysfunction in diabetes mellitus. J Int Med Res 2017, 45(6):1901-1929.

15. Yamazaki D, Hitomi H, Nishiyama A. Hypertension with diabetes mellitus complications. Hypertens Res 2018, 41(3):147-156.

16. Assar ME, Angulo J, Rodriguez-Manas L. Diabetes and ageing-induced vascular inflammation. J Physiol. 2016;594(8):2125-46.

17. Wong NKP, Nicholls SJ, Tan JTM, Bursill CA. The Role of High-Density Lipoproteins in Diabetes and Its Vascular Complications. Int J Mol Sci 2018, 19(6).

18. Mitchell AJ, Roediger B, Weninger W. Monocyte homeostasis and the plasticity of inflammatory monocytes. Cell Immunol. 2014;291(1-2):22-31.

19. Mildner A, Marinkovic G, Jung S. Murine Monocytes: Origins, Subsets, Fates, and Functions. Microbiol Spectr 2016, 4(5).

20. Shi C, Pamer EG. Monocyte recruitment during infection and inflammation. Nat Rev Immunol. 2011;11(11):762-74.

21. Garre JM, Yang G. Contributions of monocytes to nervous system disorders. J Mol Med (Berl). 2018;96(9):873-83.

22. Guilliams M, Mildner A, Yona S. Developmental and Functional Heterogeneity of Monocytes. Immunity 2018, 49(4):595-613.

23. Jaipersad AS, Lip GY, Silverman S, Shantsila E. The role of monocytes in angiogenesis and atherosclerosis. J Am Coll Cardiol. 2014;63(1):1-11.

24. Naidoo V, Naidoo M, Ghai M. Cell- and tissue-specific epigenetic changes associated with chronic inflammation in insulin resistance and type 2 diabetes mellitus. Scand J Immunol. 2018;88(6):e12723.

25. van Diepen JA, Thiem K, Stienstra R, Riksen NP, Tack CJ, Netea MG. Diabetes propels the risk for cardiovascular disease: sweet monocytes becoming aggressive? Cell Mol Life Sci. 2016;73(24):4675-84.

26. Wang H, Chen Y, Sun G, Jia P, Qian H, Sun Y. Validity of cardiometabolic index, lipid accumulation product, and body adiposity index in predicting the risk of hypertension in Chinese population. Postgrad Med. 2018;130(3):325-33.

27. Li Z, Guo X, Zheng L, Yang H, Sun Y. Grim status of hypertension in rural China: results from Northeast China Rural Cardiovascular Health Study 2013. J Am Soc Hypertens. 2015;9(5):358-64. 
28. Wang H, Chen Y, Guo X, Chang Y, Sun Y. Usefulness of cardiometabolic index for the estimation of ischemic stroke risk among general population in rural China. Postgrad Med. 2017;129(8):834-41.

29. Hall DM, Cole TJ. What use is the BMI? Arch Dis Child. 2006;91(4):283-6.

30. Ganjali S, Gotto AM Jr, Ruscica M, Atkin SL, Butler AE, Banach M, et al. Monocyte-to-HDL-cholesterol ratio as a prognostic marker in cardiovascular diseases. J Cell Physiol. 2018;233(12):9237-46.

31. Dzau VJ, Balatbat CA. Future of Hypertension. Hypertension 2019, 74(3):450-457.

32. American Diabetes A. Diagnosis and classification of diabetes mellitus. Diabetes Care. 2014;37(Suppl 1):81-90.

33. Murphy AJ, Woollard KJ, Hoang A, Mukhamedova N, Stirzaker RA, McCormick SP, et al. High-density lipoprotein reduces the human monocyte inflammatory response. Arterioscler Thromb Vasc Biol. 2008;28(11):2071-7.

34. Inonu Koseoglu H, Pazarli AC, Kanbay A, Demir O. Monocyte Count/HDL Cholesterol Ratio and Cardiovascular Disease in Patients With Obstructive Sleep Apnea Syndrome: A Multicenter Study. Clin Appl Thromb Hemost. 2018;24(1):139-44.

35. Wang HY, Shi WR, Yi X, Zhou YP, Wang ZQ, Sun YX. Assessing the performance of monocyte to highdensity lipoprotein ratio for predicting ischemic stroke: insights from a population-based Chinese cohort. Lipids Health Dis. 2019;18(1):127.

36. Chen YD, Jeng CY, Reaven GM. HDL metabolism in diabetes. Diabetes Metab Rev 1987, 3(3):653668.

37. Bekkering S, Blok BA, Joosten LA, Riksen NP, van Crevel R, Netea MG. In Vitro Experimental Model of Trained Innate Immunity in Human Primary Monocytes. Clin Vaccine Immunol. 2016;23(12):926-33.

38. Geissmann F, Manz MG, Jung S, Sieweke MH, Merad M, Ley K. Development of monocytes, macrophages, and dendritic cells. Science. 2010;327(5966):656-61.

39. Bieber K, Autenrieth SE. Insights how monocytes and dendritic cells contribute and regulate immune defense against microbial pathogens. Immunobiology 2015, 220(2):215-226.

40. Gren ST, Grip O. Role of Monocytes and Intestinal Macrophages in Crohn's Disease and Ulcerative Colitis. Inflamm Bowel Dis. 2016;22(8):1992-8.

41. Kratofil RM, Kubes P, Deniset JF. Monocyte Conversion During Inflammation and Injury. Arterioscler Thromb Vasc Biol 2017, 37(1):35-42.

42. Curat CA, Miranville A, Sengenes C, Diehl M, Tonus C, Busse R, et al. From blood monocytes to adipose tissue-resident macrophages: induction of diapedesis by human mature adipocytes. Diabetes. 2004;53(5):1285-92.

43. Oh DY, Morinaga $\mathrm{H}$, Talukdar S, Bae EJ, Olefsky JM. Increased macrophage migration into adipose tissue in obese mice. Diabetes. 2012;61(2):346-54.

44. Rao X, Zhong J, Sun Q. The heterogenic properties of monocytes/macrophages and neutrophils in inflammatory response in diabetes. Life Sci. 2014;116(2):59-66. 
45. Lehrke M, Reilly MP, Millington SC, Iqbal N, Rader DJ, Lazar MA. An inflammatory cascade leading to hyperresistinemia in humans. PLoS Med. 2004;1(2):e45.

46. Barrett TJ, Distel E, Murphy AJ, Hu J, Garshick MS, Ogando Y, et al. Apolipoprotein Al) Promotes Atherosclerosis Regression in Diabetic Mice by Suppressing Myelopoiesis and Plaque Inflammation. Circulation 2019, 140(14):1170-1184.

47. Kanter JE, Hsu CC, Bornfeldt KE. Monocytes and Macrophages as Protagonists in Vascular Complications of Diabetes. Front Cardiovasc Med. 2020;7:10.

48. Barrett TJ, Murphy AJ, Goldberg IJ, Fisher EA. Diabetes-mediated myelopoiesis and the relationship to cardiovascular risk. Ann N Y Acad Sci. 2017;1402(1):31-42.

49. Khumaedi Al, Purnamasari D, Wijaya IP, Soeroso Y. The relationship of diabetes, periodontitis and cardiovascular disease. Diabetes Metab Syndr. 2019;13(2):1675-8.

50. Groh L, Keating ST, Joosten LAB, Netea MG, Riksen NP. Monocyte and macrophage immunometabolism in atherosclerosis. Semin Immunopathol. 2018;40(2):203-14.

51. Badimon L, Vilahur $G$. Thrombosis formation on atherosclerotic lesions and plaque rupture. J Intern Med. 2014;276(6):618-32.

52. Moore KJ, Sheedy FJ, Fisher EA. Macrophages in atherosclerosis: a dynamic balance. Nat Rev Immunol. 2013;13(10):709-21.

53. Linton MRF, Yancey PG, Davies SS, Jerome WG, Linton EF, Song WL, et al: The Role of Lipids and Lipoproteins in Atherosclerosis. In: Endotext. edn. Edited by Feingold KR, Anawalt B, Boyce A, Chrousos G, Dungan K, Grossman A, Hershman JM, Kaltsas G, Koch C, Kopp P et al. South Dartmouth (MA); 2000.

54. Woollard KJ, Geissmann F. Monocytes in atherosclerosis: subsets and functions. Nat Rev Cardiol. 2010;7(2):77-86.

55. Drechsler M, Duchene J, Soehnlein O. Chemokines control mobilization, recruitment, and fate of monocytes in atherosclerosis. Arterioscler Thromb Vasc Biol. 2015;35(5):1050-5.

56. Yilmaz M, Kayancicek H. A New Inflammatory Marker: Elevated Monocyte to HDL Cholesterol Ratio Associated with Smoking. J Clin Med 2018, 7(4).

57. Akter S, Goto A, Mizoue T. Smoking and the risk of type 2 diabetes in Japan: A systematic review and meta-analysis. J Epidemiol. 2017;27(12):553-61.

58. Pencina MJ, D'Agostino RB, Sr., D'Agostino RB Jr, Vasan RS. Evaluating the added predictive ability of a new marker: from area under the ROC curve to reclassification and beyond. Stat Med. 2008;27(2):157-72. discussion 207- 112.

\section{Figures}


All the ellgible permanent residents $\geqslant 35$ years of age from each village were invited to participate in NCRCHS $(n=11,956)$

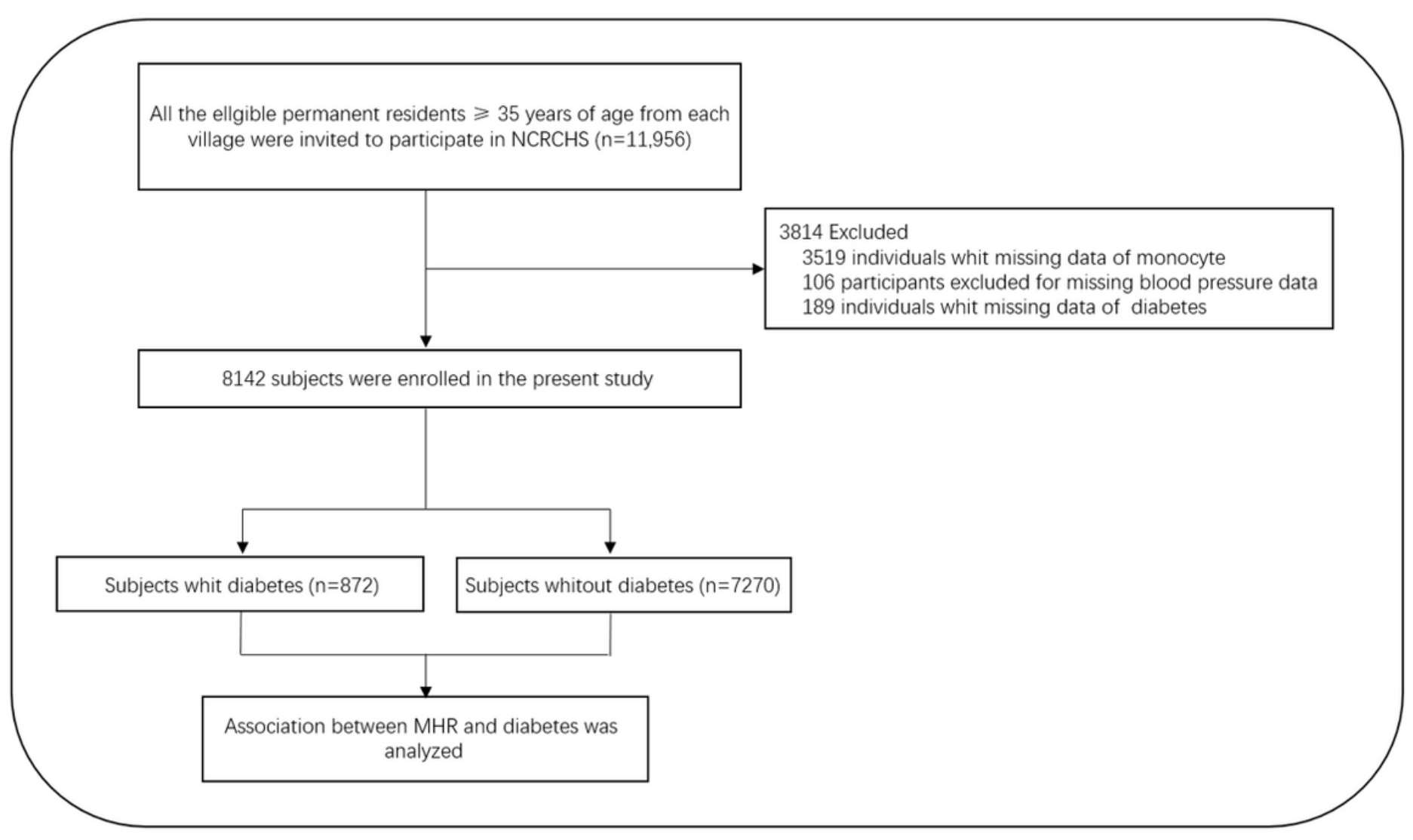

Figure 2

Flowchart describing the selection process and derivation of the study population 


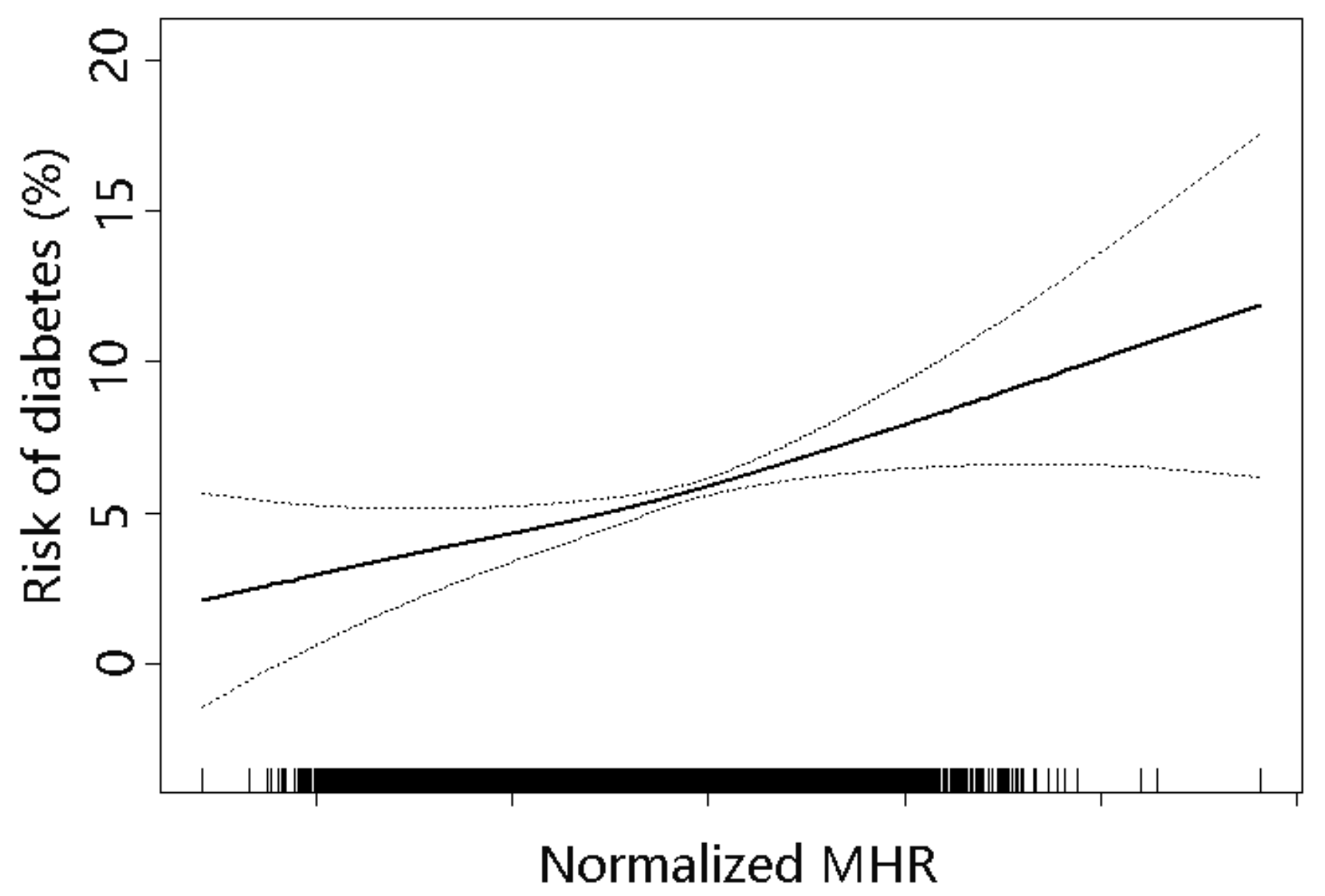

Figure 4

Smooth curve fitting was performed using generalized additive model to explore the association between MHR and the risk of diabetes after adjusting for age, sex, education level, family annual income level, physical activity, current smoking, current drinking, family history of diabetes, body mass index and systolic blood pressure. In this figure, the solid line indicates the estimated risk of diabetes while the dotted lines serve as pointwise $95 \%$ confidence intervals 